\title{
THE CENTER FOR ASTROPHYSICS REDSHIFT SURVEY:
}

\section{LUMINOSITY FUNCTION AND \\ TWO-POINT CORRELATION FUNCTION}

\author{
Valérie de Lapparent, Margaret J. Geller, and John P. Huchra, \\ Harvard-Smithsonian Center for Astrophysics \\ 60 Garden Street \\ Cambridge, MA 02138, USA
}

We compare the luminosity function and the two-point spatial correlation function for the two slices ${ }^{1}$ of the CfA redshift survey extension to $m_{B(0)} \leq 15.5\left[8^{h} \leq\right.$ R.A. $\leq 17^{h}$ and $26.5^{\circ} \leq$ DEC. $\leq 38.5^{\circ}$, with those for the early survey ${ }^{2}$ limited to $m_{B(0)} \leq 14.5\left[b^{\mathrm{II}} \geq 40^{\circ}\right.$, and DEC. $\left.\geq 0^{\circ}\right]$. The derived properties of the two samples agree within the errors. The parameters of the luminosity function are $M^{*}=-19.1 \pm 0.1$ and $\alpha=-1.2 \pm 0.1$ for the 15.5 sample, and $M^{*}=-19.3 \pm 0.1$ and $\alpha=-1.1 \pm 0.1$ for the 14.5 sample. We use an inhomogeneity-independent method to calculate the luminosity function. ${ }^{3,4}$ The slopes of the correlation functions for the two samples are $-1.5 \pm 0.35,^{5}$ shallower than the canonical slope of -1. . $^{6}$ The correlation lengths are $7.5 \pm 5 h^{-1} \mathrm{Mpc},{ }^{5}$ larger than the correlation length of $5 h^{-1} \mathrm{Mpc}$ matched to the theoretical models. ${ }^{7,8,9}$ Because of the $25 \%$ uncertainty in the mean density of the $\mathbf{1 5 . 5}$ sample, the correlation function is indeterminate on scales larger than $\sim 20 h^{-1} \mathrm{Mpc}$.

${ }^{1}$ Geller, M. J., Huchra, J. P., and de Lapparent, V. 1987, in Observational Cosmology (A. Hewitt et al. editors), IAU Symp. 124 (Dordrecht: D. Reidel), 301.

${ }^{2}$ Huchra, J., Davis, M., Latham, D., and Tonry, J. 1982, Ap. J. Suppl., 52, 89.

${ }^{3}$ Lynden-Bell, D. 1971, Mon. Not. Roy. Astr. Soc., 155, 95.

4 Turner, E. L. 1979, Ap. J., 231, 645.

5 de Lapparent, V., Geller, M. J., and Huchra, J. P. 1987, Ap. J., submitted.

${ }^{6}$ Davis, M. and Peebles, P. J. E. 1983, Ap. J., 267, 465.

7 Frenk, C. S., White, S. D. M., and Davis, M. 1983, Ap. J., 271, 417.

${ }^{8}$ Centrella, J., and Melott, A. L. 1983, Nature, 305, 196.

${ }^{9}$ Davis, M., Efstathiou, G., Frenk, C. S., and White, S. D. M. 1985, Ap. J., 292, 371. 